\title{
Temporal dynamics of hydrological threshold events
}

\author{
G. S. McGrath ${ }^{1}$, C. Hinz ${ }^{1}$, and M. Sivapalan ${ }^{2}$ \\ ${ }^{1}$ School of Earth and Geographical Sciences, University of Western Australia, Crawley, Australia \\ ${ }^{2}$ Departments of Geography \& Civil \& Environmental Engineering, University of Illinois at Urbana-Champaign, USA
}

Received: 14 July 2006 - Published in Hydrol. Earth Syst. Sci. Discuss.: 1 September 2006

Revised: 6 February 2007 - Accepted: 6 February 2007 - Published: 26 February 2007

\begin{abstract}
The episodic nature of hydrological flows such as surface runoff and preferential flow is a result of the nonlinearity of their triggering and the intermittency of rainfall. In this paper we examine the temporal dynamics of threshold processes that are triggered by either an infiltration excess (IE) mechanism when rainfall intensity exceeds a specified threshold value, or a saturation excess (SE) mechanism governed by a storage threshold. We use existing and newly derived analytical results to describe probabilistic measures of the time between successive events in each case, and in the case of the SE triggering, we relate the statistics of the time between events (the inter-event time, denoted IET) to the statistics of storage and the underlying water balance. In the case of the IE mechanism, the temporal dynamics of flow events is found to be simply scaled statistics of rainfall timing. In the case of the SE mechanism the time between events becomes structured. With increasing climate aridity the mean and the variance of the time between SE events increases but temporal clustering, as measured by the coefficient of variation (CV) of the IET, reaches a maximum in deep stores when the climatic aridity index equals 1 . In very humid and also very arid climates, the temporal clustering disappears, and the pattern of triggering is similar to that seen for the IE mechanism. In addition we show that the mean and variance of the magnitude of SE events decreases but the CV increases with increasing aridity. The CV of IETs is found to be approximately equal to the $\mathrm{CV}$ of the magnitude of $\mathrm{SE}$ events per storm only in very humid climates with the CV of event magnitude tending to be much larger than the $\mathrm{CV}$ of IETs in arid climates. In comparison to storage the maximum temporal clustering was found to be associated with a maximum in the variance of soil moisture. The CV of the time till the first saturation excess event was found to be greatest when the initial storage was at the threshold.
\end{abstract}

Correspondence to: Christoph Hinz

chinz@cyllene.uwa.edu.au

\section{Introduction}

Many rapid hydrological processes such as runoff (Horton, 1933; Dunne, 1978), preferential flow (Beven and Germann, 1982), and erosion (Fitzjohn et al., 1998), are not continuous, but are triggered by thresholds. For example surface runoff occurs when the rainfall intensity is greater than the soils ability to adsorb it via infiltration. As a result some of these processes may even cease or are not even triggered if the rainfall event is too small. Because we consider rapid flow processes the duration of flow events is often small in comparison to the time between rainfall events. Therefore, what results from the threshold triggering, when we look at a time series, is a sequence of discrete, episodic events.

As the occurrence of these episodic processes is linked to the timing and magnitude of rainfall events it is natural therefore, to consider improving our understanding of how the temporal occurrence of these flow events relates to the structure of rainfall. This is the focus of this paper.

The temporal dynamics of preferential flow triggering due to between-storm and within-storm rainfall variability has been previously explored via the numerical simulation approach, using synthetic rainfall time series (Struthers et al., 2007a,b). Struthers et al. (2007b) were able to relate aspects of the probability distribution function (pdf) and specific statistical characteristics of the storm inputs to statistical properties and aspects of the pdfs of preferential flow and runoff magnitude and timing. The analysis was not able to separate the contributions of the various runoff mechanisms to the statistical properties of the resulting temporal flow dynamics. A simpler, more general approach was required to further develop the ideas presented in this work.

There are two triggers which are shared by a number of processes. Some processes are triggered, as already mentioned, by a rainfall intensity threshold. Examples include macropore flow through soil (Beven and Germann, 1982; Heppell et al., 2002), and surface runoff (Horton, 1933). This triggering is termed infiltration excess. Some processes are triggered by a storage threshold or storm amount. These include various types of preferential flow (Beven and Germann,

Published by Copernicus GmbH on behalf of the European Geosciences Union. 
1982; Kung, 1990; Haria et al., 1994; Wang et al., 1998; Bauters, 2000; Dekker et al., 2001; Heppell et al., 2002), interception (Crockford and Richardson, 2000; Zeng et al., 2000), and hillslope outflow through subsurface flow pathways (Whipkey, 1965; Mosley, 1976; Uchida et al., 2005; Tromp-van Meerveld and McDonnell, 2006). The storage threshold will be referred to here as saturation excess, although the use of this term is not often associated with some of the above processes.

In this article we examine the simplest possible conceptualizations for these two triggers. For infiltration excess we will use a threshold rainfall intensity (Horton, 1933; Heppell et al., 2002; Kohler et al., 2003) neglecting any soil moisture storage controls on the infiltration capacity. For saturation excess we use the opposite extreme, a threshold storage, depending only on the storm amount and not its intensity. While these two conceptualisations are very simplistic we expect a mixture of the results from these two processes to reflect more complex triggers, where triggering is a function of both rainfall intensity and storage.

The saturation excess mechanism captures the carry over of storage from one rainfall event to the next. As a result there is an enhanced probability that a second flow event will occur shortly after a flow event has just occurred because storage is more likely to be nearer the threshold in this interval. We hypothesize that this leads to temporal clustering of saturation excess, which means that multiple events occur in short periods of time separated by longer event-free intervals.

A second aspect of the paper is to address the issue of observability. For some processes we cannot measure directly the flux. Preferential flow is one such example. What we can observe of preferential flow is the timing of episodic pesticide leaching events (Hyer et al., 2001; Fortin et al., 2002; Laabs et al., 2002; Kjær et al., 2005) which are driven by this process. We also know that preferential flow is related to thresholds in soil moisture (Beven and Germann, 1982) and this can be measured reasonably well. For other processes, like subsurface hillslope outflow via soil pipes, the flux and timing might be observable but the internal, storage process much less so. There is clearly a need to better understand the inter-relationships between timing, flux, and storage in order to be able to make better predictions of these threshold processes where the internal dynamics are largely hidden (Rundle et al., 2006, this issue). In this paper it will be possible to make this inter-comparison for the saturation excess trigger.

The paper begins with a brief overview of the simple models of rainfall adopted for this analysis. Based upon this we derive analytically the statistics of the time between threshold events for infiltration excess and saturation excess mechanisms, and for saturation excess we also present existing and newly derived statistics of the runoff flux based upon the original work of Milly $(1993 ; 2001)$. Finally, we explore the results in the context of storm properties and the climate setting.

\section{Rainfall models}

For modelling purposes we adopt simple stationary descriptions of rainfall without any seasonal dependence. Storms are characterised only by three parameters, their total depth $h[\mathrm{~L}]$, a maximum within-storm intensity $I_{\max }[\mathrm{L} / \mathrm{T}]$, and a time between storms $t_{b}$ [T]. Storms are considered to be instantaneous events, independent of one another, therefore satisfying the Poisson assumption as used commonly in hydrology (Milly, 1993; Rodriguez-Iturbe et al., 1999). Such an assumption is considered valid at near daily time scales (Rodriguez-Iturbe and Isham, 1987).

As we are primarily concerned with an event based description of processes, this rainfall model is appropriate to make direct comparisons between the process and its driver. Our objective is to capture the inter-(rainfall)-event dynamics, i.e. this event did or did not trigger the threshold and not the detail of within-event processes which is left for future research.

The Poisson assumption implies that the random time between storms, the inter-storm time, that results is described by an exponential probability density function (pdf) (Rodriguez-Iturbe et al., 1999):

$$
g_{T b}\left[t_{b}\right]=\frac{1}{\bar{t}_{b}} e^{-t_{b} / \bar{t}_{b}}
$$

which is fully characterised by its mean $\bar{t}_{b}[\mathrm{~T}]$. Storm depths are also assumed to follow an exponential pdf $f_{H}[h]$ with a mean $\bar{h}[\mathrm{~L}]$. The maximum within-storm rainfall intensity is also considered to be exponentially distributed with a mean of $\bar{I}_{\max }[\mathrm{L} / \mathrm{T}]$.

Clearly this rainfall model is a gross simplification of real rainfall, particularly as it neglects seasonality. However its advantage is it makes the later derivations analytically tractable. Considering it's use to describe within a season rainfall may be more appropriate, but the statistics we will derive later may not necessarily reflect the transient dynamics of storage in certain climates (Rodriguez-Iturbe et al., 2001). We also derive here statistics based upon an arbitrary initial condition which may account for this transient behaviour.

\section{Statistics of temporal dynamics}

Based on the rainfall signal and soil properties we would like to quantify the probability that a given flow event, involving the exceedance of some kind of threshold, occurs. We use the time between events as the random variable that characterises this event probability. This concept is illustrated for a rainfall intensity threshold (Fig. 1a) and a soil moisture storage threshold (Fig. 1b). The random time to reach these thresholds for the first time is referred to as a first passage time (FPT) denoted as $\tau_{1}$, and the time between flow events is referred to as the inter-event time (IET) denoted as $\tau_{2}$ and $\tau_{3}$ in 


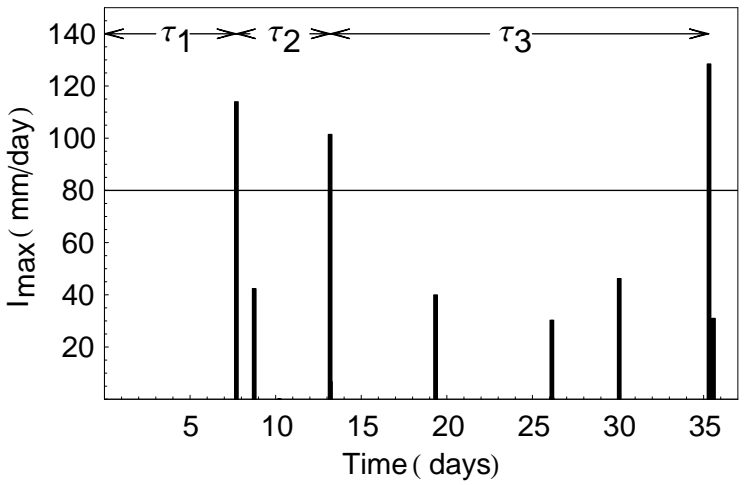

(a)

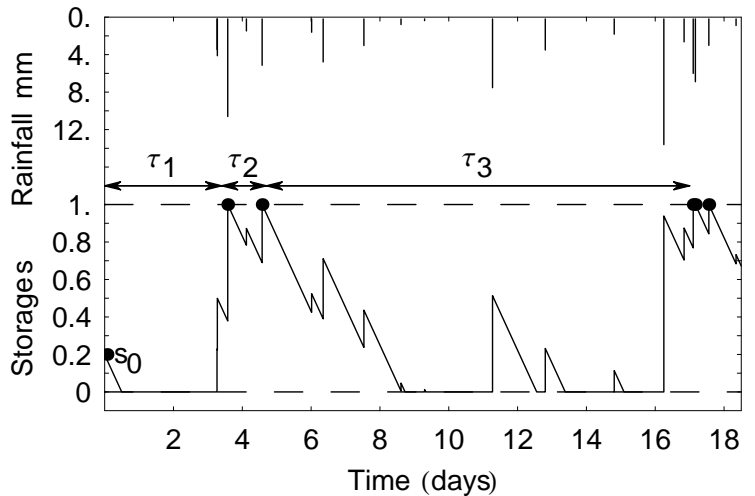

(b)

Fig. 1. Definition of the more general first passage time $\tau_{1}$ and the inter-event time $\tau_{2}$ and $\tau_{3}$ for (a) a threshold rainfall intensity infiltration excess (IE) trigger, where in this example intensities above $I_{\xi}=80 \mathrm{~mm} /$ day trigger an IE event; and (b) a storage, or saturation excess mechanism (SE), occurring when $s=1$. The variable $s_{0}$ denotes the initial storage.

Fig. 1a and Fig. 1b. In the case of infiltration excess runoff, a flow event is triggered when the maximum rainfall intensity within a storm exceeds the infiltration capacity $I_{\xi}$. Similalry, when the soil water storage reaches a critical capacity a saturation excess event is deemed to have been triggered.

To quantify the pdfs of the first passage times and the IETs, we use the first four central moments. For completeness we define the moments in this section and will derive analytical expressions for them in Sect. 5.2 and A. The mean $T_{\mu}[\mathrm{T}]$ and the $k^{\text {th }}$ central moment $\mu_{k}$ of the random variable $\tau$ are related to the pdf $g_{\mathrm{T}}[\tau]$ by the following (Papoulis, 2001):

$$
\begin{gathered}
T_{\mu}=E[\tau]=\int_{-\infty}^{\infty} \tau g_{\mathrm{T}}[\tau] \mathrm{d} \tau \\
\mu_{k}=E\left[(\tau-E[\tau])^{k}\right]=\int_{-\infty}^{\infty}\left(\tau-T_{\mu}\right)^{\mathrm{k}} g_{\mathrm{T}}[\tau] \mathrm{d} \tau
\end{gathered}
$$

for integers $k \geq 2$, where $E$ [ ] denotes the expectation operator. In addition to the mean and the variance $T_{\sigma^{2}}=\mu_{2}$ $\left[\mathrm{T}^{2}\right]$, we will also use the dimensionless statistic, the coefficient of variation $(\mathrm{CV}), T_{c v}=\sqrt{T_{\sigma^{2}}} / T_{\mu}[-]$, the ratio of
Table 1. Storm and infiltration excess inter-event statistics

\begin{tabular}{lcc}
\hline Statistic & Storm & Infiltration excess \\
\hline$T_{\mu}$ & $\bar{t}_{b}$ & $\bar{t}_{b} e^{I_{\xi} / \bar{I}_{\max }}$ \\
$T_{\sigma^{2}}$ & $\bar{t}_{b}^{2}$ & $\left(\bar{t}_{b} e^{I_{\xi} / \bar{I}_{\max }}\right)^{2}$ \\
$T_{c v}$ & 1 & 1 \\
$T_{\varepsilon}$ & 2 & 2 \\
$T_{\kappa}$ & 6 & 6 \\
\hline
\end{tabular}

the standard deviation to the mean, which gives a measure of the variability relative to the mean; the coefficient of skewness $T_{\varepsilon}=\mu_{3} / \mu_{2}{ }^{3 / 2}$ [-], which describes the asymmetry of the probability distribution, where positive values indicate that the distribution has a longer tail towards larger values than smaller values; and the coefficient of kurtosis $T_{\kappa}=\mu_{4} / \mu_{2}^{2}-3[-]$, where positive values indicate a more peaked distribution, in comparison to a normally distributed variable, and "fatter tails" i.e. an enhanced probability of extreme values (Papoulis, 2001).

In order to compare the statistical properties of flow event triggers with the rainfall signal, we summarise here the rainfall in terms of IET statistics. The idea here is to compare and contrast storm IET and flow IET statistics for the various threshold driven processes. Events which are temporally independent of one another have an exponential IET pdf. Table 1 lists the statistics for the storm IET.

A property of the exponential distribution is that the mean is equal to the standard deviation, and therefore the $\mathrm{CV}$ is equal to 1 . The $\mathrm{CV}$ of the IET $T_{c v}$ is often used to distinguish temporally clustered and unclustered processes (Teich et al., 1997). Temporal clustering is said to occur when $T_{c v}>1$, a $T_{c v}=0$ indicates no variability and exactly regular events, while a $T_{c v}<1$ may indicate a quasi-periodic process (Wood et al., 1995; Godano et al., 1997).

\section{Infiltration excess inter-event time statistics}

Here we assume for simplicity that the infiltration capacity $I_{\xi}$ is constant (Heppell et al., 2002; Kohler et al., 2003), and therefore soil moisture controls on the infiltration capacity are considered insignificant. This approach provides an extreme contrast to the saturation excess mechanism considered. It may actually better represent well more extreme rainfall, where the antecedent soil moisture has little impact on event triggering. For example Heppell et al. (2002) classified preferential flow events in a clay loam soil as antecedent soil moisture limited and non-antecedent limited events. The non-antecedent limited events were related to storms where the maximum within-storm rainfall intensity was much larger than the mean storm intensity. 
This constant threshold filtering is the same as the rainfall filtering described in Rodriguez-Iturbe et al. (1999) in the context of the soil water balance. They showed that a threshold filtering of the depth of rainfall with Poisson arrivals resulted in a new Poisson process. The rate of events over this threshold equalled the storm arrival rate multiplied by the probability of exceeding the threshold storm depth by a single event (Rodriguez-Iturbe et al., 1999). The difference for the IE trigger considered here lies only in semantics. When the maximum within-storm rainfall intensity $I_{\max }$ is exponentially distributed with mean $\bar{I}_{\max }$ the probability that $I_{\max }>I_{\xi}$ is equal to $e^{-I_{\xi} / \bar{I}_{\max }}$ and therefore the resulting mean time between IE events is equal to $T_{\mu}^{I}=\bar{t}_{b} e^{I_{\xi} / \bar{I}_{\max }}$. Table 1 lists the IET statistics that result.

A soil with $I_{\xi}=2 \bar{I}_{\max }$ results in a mean IET which is $e^{2}$ times longer than the mean storm IET $\bar{t}_{b}$, and a variance $e^{4}$ times greater than the storm IET variance $\bar{t}_{b}^{2}$. The higher central moments remain unchanged. The IE filtering therefore results in temporal dynamics that are statistically the same as the rainfall's, but scaled by a factor related to the single event probability of exceeding the threshold. This is illustrated in Fig. 2a, which is a semi-log plot of an example infiltration excess IET pdf, corresponding to the above example, shown in comparison to the rainfall IET pdf.

\section{Saturation excess filtering}

In this section saturation excess is described on the basis of Milly's (1993) nonlinear storage-runoff model. Milly (1994) used this model to describe the impact of rainfall intermittency and soil water storage on runoff generation at catchment scales, and which he successfully used to capture much of the spatial variability in the annual water balance in catchments across much of continental USA. We use this minimalist framework to model the triggering of runoff by the exceedance of a threshold value of storage. We first review his analytical results for the statistics of soil moisture storage and the mean water balance components. We then go on to derive from these results the variance of the saturation excess runoff flux on a per storm basis. We will later relate these statistics to those of the temporal dynamics of saturation excess triggering.

\subsection{Storage and the water balance}

Milly's (1993) water balance model is represented in terms of a simple bucket with a fixed storage capacity $w_{0}[\mathrm{~L}]$ which wets in response to random storm events and dries in the inter-storm period, of random duration, due to a constant evaporative demand $E_{m}[\mathrm{~L} / \mathrm{T}]$. The threshold soil moisture $\left(s_{\xi}=1[-]\right)$ for flow initiation is assumed to have been reached when the store is filled to capacity. Any excess rainfall becomes saturation excess. The resulting stochastic balance equation for water storage $s[-]$ (a dimensionless storage nor- malised by $w_{0}$ ) is:

$$
\frac{\mathrm{d} s}{\mathrm{~d} t}=-L[s]+F[s, t]
$$

where $t[\mathrm{~T}]$ denotes time, $L[s]\left[\mathrm{T}^{-1}\right]$ the (normalised by $w_{0}$ ) evaporative losses from storage. $F[s, t][-]$ denotes instantaneous random infiltration events (normalised by $w_{0}$ ), occurring at discrete times $t_{i}$. Infiltration is limited by the available storage capacity i.e. $F\left[s, t_{i}\right]=\min \left[1-s_{i}^{-}, h_{i} / w_{0}\right]$, where $s_{i}^{-}[-]$denotes the antecedent soil moisture and $h_{i}$ [L] the random storm depth. Evaporation losses are given by:

$$
L[s]=\left\{\begin{array}{llr}
0 & \text { for } & s=0 \\
\frac{E_{m}}{\mathrm{w}_{0}} & \text { for } & 0<s \leq 1
\end{array}\right.
$$

In this article we largely consider the transformation of Eq. (4) directly into the statistical properties of IETs, however, we discuss briefly here how to numerically simulate a storage time series. This is done in part to help explain the conceptualisation of the process as well as to describe how we generated example time series presented in Sect. 6.3.1. Simulation involves generating a random storm IET $t_{b}$ and a subsequent storm depth $h$ from their respective probability distributions. Storage at any time $t$ in the inter-storm period can be calculated from $s[t]=\max \left[0, s_{0}-E_{m}\left(t-t_{0}\right) / w_{0}\right]$, where $t_{0}$ is the time of the last storm when soil moisture was $s_{0}$. At the end of the inter-storm period $t_{i}=t_{0}+t_{b}$ storage immediately prior to the storm is given by $s_{i}^{-}$. Storage increases from $s_{i}^{-}$to $s_{i}^{+}=\min \left[1, s_{i}^{-}+h / w_{0}\right]$ due to the storm also at a time $t_{i}$. This occurs as an instantaneous event with storage taking on two separate values immediately either side of time $t_{i}$. The simulation is then continued with $s_{i}^{+}$as the new $s_{0}$ and so on.

From the above model and the characteristics of rainfall presented before, two similarity parameters can be defined: the supply ratio $\alpha=w_{0} / \bar{h}$, the ratio of storage capacity to mean storm depth, and the demand ratio $\beta=w_{0} /\left(E_{m} \bar{t}_{b}\right)$, the ratio of storage capacity to mean potential inter-storm evaporation (Milly, 1993). When $\alpha=0$ the rainfall supply is infinitely larger than storage capacity and when $\alpha=\infty$ the supply is negligible compared to the amount in storage at capacity. Similarly $\beta=0$ indicates infinite evaporative demand and $\beta=\infty$ negligible demand relative to the storage capacity. Typically one could expect the parameters to range from about 1 to 100 depending upon the process considered. For example a thin, near surface, water repellent layer with $10 \mathrm{~mm}$ of storage could have realistic $\alpha$ values of 1 to 10 depending upon the mean storm depth. Runoff controlled by storage throughout the rooting depth may have much larger values (Milly, 2001).

The ratio $A I=\left(E_{m} \bar{t}_{b}\right) / \bar{h}=\alpha / \beta$ defines the relative balance between mean potential inter-storm evaporation and the mean storm depth and is otherwise known as the climatic 
aridity index (Budyko, 1974; Arora, 2002). An $A I<1$ describes a humid climate, $A i>1$ an arid climate and $A I=1$ defines equal mean rainfall and mean potential evaporation.

Milly (1993) derived the pdf of water storage in the form of two derived distributions of storage: one for a time immediately before a storm $f_{S^{-}}$, the antecedent storage pdf, and one immediately after a storm $f_{S^{+}}$. The pdf for storage for all times $f_{S}$, that resulted from that analysis was found to be equal to $f_{S^{-}}$(Milly, 2001). This was essentially because storms were modelled as instantaneous events, with no duration, and the expected time of the next storm is an exponential distribution emanating from a memoryless Poisson process for the rainfall arrivals. The pdf of $f_{S}$ is given by (Milly, 2001):

$$
f_{S}=p \beta e^{(\alpha-\beta) s}+q \delta[s]
$$

where $\delta$ denotes the Dirac delta function, $p$ describes the probability storage is at capacity $(s=1)$, and $q$ the probability that the soil is dry $(s=0)$ and are given by:

$$
\begin{aligned}
& p=\frac{\alpha-\beta}{\alpha e^{\alpha-\beta}} \\
& q=\frac{\beta-\alpha}{\beta e^{\beta-\alpha}-\alpha}
\end{aligned}
$$

The resulting mean $S_{\mu}[-]$ and variance $S_{\sigma^{2}}$ [-] of storage are given by Eq. (8) and Eq. (9) respectively (Milly, 2001):

$$
\begin{gathered}
S_{\mu}=\frac{1}{\alpha-\beta}+\frac{1+\beta e^{\beta-\alpha}}{\beta e^{\beta-\alpha}-\alpha} \\
S_{\sigma^{2}}=\frac{1}{(\alpha-\beta)^{2}}-\frac{1+(\alpha+2) \beta e^{\beta-\alpha}}{\left(\beta e^{\beta-\alpha}-\alpha\right)^{2}}
\end{gathered}
$$

Based on the definition of the expected value of a function $g[x]$ of a random variable $x$, with a known pdf $f_{X}[x]$ (Papoulis, 2001):

$$
E[g[x]]=\int_{-\infty}^{\infty} g[x] f_{X}[x] \mathrm{d} x
$$

Milly (1993) derived the mean actual evaporation $E_{a}[\mathrm{~L}]$ per inter-storm period via:

$$
\frac{E_{a}}{\bar{h}}=\frac{w_{0}}{\bar{h}} \int_{0^{-}}^{1} L[s] f_{S}[s] \mathrm{d} s=1-\frac{\alpha-\beta}{\alpha e^{\alpha-\beta}-\beta}
$$

where the $0^{-}$is used to denote inclusion of the probability that $s=0$.

From mass balance considerations Milly (1993) then determined the mean runoff per storm $Q_{\mu}$ [L] as follows:

$$
\frac{Q_{\mu}}{\bar{h}}=1-\frac{E_{a}}{\bar{h}}=\frac{\alpha-\beta}{\alpha e^{\alpha-\beta}-\beta}
$$

Extending such analysis, we derive here for the first time the variance of flux per storm event $Q_{\sigma^{2}}\left[\mathrm{~L}^{2}\right]$ which we calculate from the definition of the variance of a function of two random variables $G[x, y]$ which have a joint pdf $f_{X Y}[x, y]$ (Papoulis, 2001) which is given by:

$$
\begin{aligned}
& E\left[(G[x, y]-E[G[x, y]])^{2}\right]= \\
& \int_{-\infty}^{\infty} \int_{-\infty}^{\infty}(G[x, y]-E[G[x, y]])^{2} f_{X Y}[x, y] \mathrm{d} x \mathrm{~d} y
\end{aligned}
$$

The magnitude of saturation excess generated on a single event $Q_{i}$ is given by:

$$
Q_{i}\left[h_{i}, s_{i}^{-}\right]=\max \left[0, h_{i}-w_{0}\left(1-s_{i}^{-}\right)\right]
$$

which depends upon the two random variables, storm depth $h_{i}$ and the antecedent soil moisture $s_{i}^{-}$. As the pdf of soil moisture immediately prior to a rainfall event $f_{S^{-}}$is equivalent to the pdf of soil moisture $f_{S}$ Eq. (6) in this instance (Milly, 2001) and as $h$ and $s^{-}$are independent random variables we can apply Eq. (13) and the condition Eq. (14) to the calculation of the saturation excess runoff variance $Q_{\sigma^{2}}$ by the following:

$$
\begin{aligned}
\frac{Q_{\sigma^{2}}}{\bar{h}^{2}} & =\int_{0^{-}}^{1} \int_{w_{0}(1-s)}^{\infty}\left(\frac{w_{0}}{\bar{h}}\left(\frac{h}{w_{0}}-1+s\right)-\frac{Q_{\mu}}{\bar{h}}\right)^{2} \times \\
& w_{0} f_{H}[h] f_{S}[s] \mathrm{d} h \mathrm{~d} s+ \\
& \int_{0^{-}}^{1} \int_{0}^{w_{0}(1-s)}\left(-\frac{Q_{\mu}}{\bar{h}}\right)^{2} f_{H}[h] w_{0} f_{S}[s] \mathrm{d} h \mathrm{~d} s \\
& =\frac{(\alpha-\beta)\left(2 \alpha e^{\alpha-\beta}-\alpha-\beta\right)}{\left(\alpha e^{\alpha-\beta}-\beta\right)^{2}}
\end{aligned}
$$

The statistics of storage (Eq. 8, Eq. 9) and the flux (Eq. 12, Eq. 15) will be explored in more detail later on.

\subsection{Statistics of saturation excess timing}

For the type of stochastic process modelled by Milly (1993), Masoliver (1987) and Laio et al. (2001) provided derivations for the mean time to reach a threshold for the first time. This methodology was subsequently applied to describe the mean duration of soil moisture persistence between upper and lower bounds in order to investigate vegetation water stress (Ridolfi et al., 2000).

The mean FPT describes the expected waiting time till an event dependent upon the initial condition, of which the mean IET is a special case. The temporal dynamics also consists of the variability about this mean behaviour, therefore in Appendix A we present, for the first time, an extension of the derivation for the mean first passage time (Laio et al., 2001) giving a general solution to the higher moments of the FPT, such as the variance.

Using equations (A5), (A13) and (A10), with $n=1, T_{0}=1$ and $L[s]$ as given by Eq. (5) we can derive the mean time 
$T_{\mu}^{s}\left[s_{0}, s_{\xi}\right]$ till an arbitrary threshold storage $s_{\xi}$ is reached for the first time, when the initial storage was $s_{0} \leq s_{\xi}$ as:

$$
\begin{array}{r}
\frac{T_{\mu}^{s}\left[s_{0}, s_{\xi}\right]}{\bar{t}_{b}}=\frac{\alpha\left(\alpha e^{s_{\xi}(\alpha-\beta)}-\beta e^{s_{0}(\alpha-\beta)}\right)}{(\alpha-\beta)^{2}}- \\
\frac{\beta\left(\alpha\left(s_{\xi}-s_{0}\right)+1\right)}{\alpha-\beta}
\end{array}
$$

This is the same result, after appropriate adjustment of parameters, as given by Eq. (31) in Laio et al. (2001). The mean saturation excess IET $T_{\mu}^{s}[1,1]$ comes from substitution of $s_{0}=s_{\xi}=1$ in Eq. (16):

$$
\frac{T_{\mu}^{s}[1,1]}{\bar{t}_{b}}=\frac{\alpha e^{\alpha-\beta}-\beta}{\alpha-\beta}
$$

As a result of our more general derivation we can use equations (A5), (A13) and (A10) together with $n=2$, and $T_{1}=T_{\mu}^{s}\left[s_{0}, s_{\xi}\right]$, to derive the raw moment $T_{2}$. From the relationship between the central moments and the raw moments (see Table A1), the FPT variance is calculated by $T_{\sigma^{2}}^{s}\left[s_{0}, s_{\xi}\right]=T_{2}-T_{1}^{2}$. After substitution of $s_{0}=s_{\xi}=1$ we can show the variance of IET arising from the storage threshold, $T_{\sigma^{2}}^{s}[1,1]$ is given by:

$$
\begin{array}{r}
\frac{T_{\sigma^{2}}^{s}[1,1]}{\bar{t}_{b}^{2}}=\frac{2 \alpha \beta e^{\alpha-\beta}(\alpha+\beta+2)}{(\beta-\alpha)^{2}}+ \\
\frac{(\alpha+\beta)\left(\beta^{2}-\alpha^{2} e^{2(\alpha-\beta)}\right)}{(\beta-\alpha)^{3}}
\end{array}
$$

In this paper we do not present the full solution for $T_{\sigma^{2}}^{s}\left[s_{0}, s_{\xi}\right]$ or the other higher moments as they are rather cumbersome; however complete solutions for the first four moments are provided as supplementary material (http://www.hydrol-earth-syst-sci.net/11/923/2007/ hess-11-923-2007-supplement.pdf). Table 2 summarises the first four central moments of the IET for three limiting cases, as discussed in more detail in the next section.

\section{Results and Discussion}

\subsection{Climate controls on threshold storage events}

This section discusses the analytical results for the statistical properties of the time between SE events and the statistics of the SE event magnitudes based on an investigation of three limiting climates: a super humid climate with an aridity in$\operatorname{dex} A I=0$ by taking the limit $\alpha \rightarrow 0$ of the derived statistics; a super arid climate with an aridity index $A I=\infty$ by taking the limit $\beta \rightarrow 0$; and an intermediate climate with $A I=1$ by taking the limit $\beta \rightarrow \alpha$. The analytical results corresponding to these limiting cases are summarised in Table 2.

\subsubsection{Super humid climates: $A I=0$}

In very humid climates the temporal statistics of saturation excess IET are identical to that of the rainfall (see Table 2). This is because with an excess supply of rainfall and negligible evaporative demand the soil is always saturated. The statistics are consistent with an inter-event time which is exponentially distributed. No filtering takes place as every rainfall event triggers flow and all rainfall becomes saturation excess (see Table 2). The rainfall signal is therefore an excellent indicator of the timing, frequency and magnitude of SE events.

\subsubsection{Super arid climates: $A I=\infty$}

The IET statistics of SE events for an arid climate (Table 2) resemble those of the IE filtering described above (refer to Table 1). The average rate of SE events is scaled by the probability of filling the store completely in a single event i.e. by $P\left[h>w_{0}\right]=e^{-w_{0} / \bar{h}}=e^{-\alpha}$. For example, consider a water repellent soil with a distribution layer which triggers finger flow after a critical water content (Dekker et al., 2001) equivalent to $10 \mathrm{~mm}$ of storage. In an extremely arid climate ( $\beta \approx 0)$ with $\bar{h}=2 \mathrm{~mm}(\alpha=5)$, and $\bar{t}_{b}=5$ days, the resulting mean and variance of the preferential flow IET is $5 e^{5}$ days and $25 e^{10}$ days $^{2}$ respectively. Again the statistics are consistent with an IET which is exponentially distributed. The independence of events is maintained because soil moisture is completely depleted before every rainfall event. In this instance a simple filtering of the rainfall, for $h>w_{0}$, provides a good predictor of the timing, relative frequency and magnitude of storage threshold flow events.

The mean saturation excess magnitude $Q_{\mu}$ is also proportional to the mean storm depth scaled by the probability of filling the store on a single event (see Table 2). Unlike the simple threshold filtering, as described by the temporal dynamics, the variance of the magnitude of saturation excess events per storm $Q_{\sigma^{2}}$ is larger than would be expected for an exponentially distributed random variable with the given mean. We believe this is due to the fact that the statistic includes a large number of zero values where storms do not trigger a threshold storage event. A CV of event magnitude $Q_{c v}=\sqrt{2 e^{\alpha}-1}$ indicates that in the limit of a very arid climate the relative variability of the magnitude of these events increases with increasing storage capacity and decreasing storm depth (increasing $\alpha$ ). For the example described above $(\alpha=5)$ the mean, variance and $\mathrm{CV}$ of event magnitudes are equal to $Q_{\mu}=0.013 \mathrm{~mm} Q_{\sigma^{2}}=0.054 \mathrm{~mm}^{2}$ and $Q_{c v}=17.2$ respectively.

\subsubsection{A balanced climate: $A I=1$}

For the case when demand balances supply $(A I=1)$, the mean saturation excess IET is $1+\alpha$ times greater than the mean storm IET $\bar{t}_{b}$ and the variance is 
Table 2. Summary statistics of the saturation excess inter-event times and event magnitudes in three limiting climates

\begin{tabular}{|c|c|c|c|c|}
\hline Statistic & Rainfall & $\begin{array}{c}\text { Humid } \\
A I=0\end{array}$ & $\begin{array}{c}\text { Intermediate } \\
\quad A I=1\end{array}$ & $\begin{array}{c}\text { Arid } \\
A I=\infty\end{array}$ \\
\hline$T_{\mu}^{s}[1,1]$ & $\bar{t}_{b}$ & $\bar{t}_{b}$ & $\bar{t}_{b}(1+\alpha)$ & $\bar{t}_{b} e^{\alpha}$ \\
\hline$T_{\sigma^{2}}^{s}[1,1]$ & $\bar{t}_{b}^{2}$ & $\bar{t}_{b}^{2}$ & $\frac{2}{3}\left(\alpha^{3}+2 \alpha^{2}+2 \alpha+1\right) \bar{t}_{b}^{2}$ & $\left(\bar{t}_{b} e^{\alpha}\right)^{2}$ \\
\hline$T_{c v}^{s}[1,1]$ & 1 & 1 & $\frac{\sqrt{3}}{\sqrt{2}} \frac{\sqrt{\alpha^{3}+2 \alpha^{2}+2 \alpha+1}}{1+\alpha} \geq 1$ & 1 \\
\hline$T_{\varepsilon}^{S}[1,1]$ & 2 & 2 & $\frac{\sqrt{3}\left(4 \alpha^{5}+20 \alpha^{4}+40 \alpha^{3}+45 \alpha^{2}+30 \alpha+10\right)}{5 \sqrt{2 \alpha(\alpha(\alpha+3)+3)+3}} \geq 2$ & 2 \\
\hline$T_{\kappa}^{s}[1,1]$ & 6 & 6 & $\frac{B_{1} \alpha^{7}+B_{2} \alpha^{6}+B_{3} \alpha^{5}+B_{4} \alpha^{4}+B_{5} \alpha^{3}+B_{6} \alpha^{2}+B_{7} \alpha+B_{8}}{35(2 \alpha(\alpha(\alpha+3)+3)+3)^{2}}-3 \geq 6^{a}$ & 6 \\
\hline$Q_{\mu}$ & $\bar{h}$ & $\bar{h}$ & $\frac{\bar{h}}{1+\alpha}$ & $\frac{\bar{h}}{e^{\alpha}}$ \\
\hline$Q_{\sigma^{2}}$ & $\bar{h}^{2}$ & $\bar{h}^{2}$ & $\frac{(1+2 \alpha)}{(1+\alpha)^{2}} \bar{h}^{2}$ & $\frac{2 e^{\alpha}-1}{e^{2 \alpha}} \bar{h}^{2}$ \\
\hline$Q_{c v}$ & 1 & 1 & $\sqrt{1+2 \alpha}$ & $\sqrt{2 e^{\alpha}-1}$ \\
\hline
\end{tabular}

${ }^{a}$ Constants $B_{i}$ are given by $B_{1}=408, B_{2}=3276, B_{3}=11214, B_{4}=22050, B_{5}=27720, \quad B_{6}=22680$, $B_{7}=11340, B_{8}=2835$

$2\left(\alpha^{3}+2 \alpha^{2}+2 \alpha+1\right) / 3$ times greater than the variance of storm IETs $\bar{t}_{b}^{2}$ (see Table 2). To put this into context using the example described above for a water repellent soil i.e. $w_{0}=10 \mathrm{~mm}, \bar{h}=2 \mathrm{~mm}$ per storm $(\alpha=5)$, and say $E_{m}=2 \mathrm{~mm} /$ day and $\bar{t}_{b}=1$ day $(\beta=5)$, results in $T_{\mu}^{s}[1,1]=6$ days, $T_{\sigma^{2}}^{s}[1,1]=124$ days $^{2}, T_{c v}^{s}[1,1]=1.86$, $T_{\varepsilon}^{s}[1,1]=644$, and $T_{\kappa}^{s}[1,1]=17.7$. In comparison the rainfall has $T_{\mu}^{r}[1,1]=1$ day, $T_{\sigma^{2}}^{r}[1,1]=1$ day, $T_{c v}^{r}[1,1]=1$, $T_{\varepsilon}^{r}[1,1]=2$, and $T_{\kappa}^{r}[1,1]=6$ (refer to Table 2). The coefficient of variation of saturation excess inter-event times, being greater than 1 , is indicative that the process is temporally clustered.

Unlike the filtering that is associated with the IE mechanism, the storage threshold filtering changes the form of the IET pdf. Figure $2 b$ shows conceptually how the saturation excess IET pdf changes from an exponential form in very humid climates (the same pdf as the rainfall IET) to a more peaked, fatter tailed distribution at $A I=1$, reverting to an exponentially distributed variable again at $A I=\infty$. Interestingly it can be seen for the parameters chosen that extreme IETs are actually more probable when $A I=1$ than when $A I=\infty$.

Referring again to the IET statistics for $A I=1$ (Table 2), as the storage capacity increases, relative to the supply and demand, (i.e. increasing $\alpha$ ) the mean storage threshold IET increases, as does the variance and this increases faster than the square of the mean, which results in an increase in the CV. Therefore the clustering of events in time tends to increase with increasing storage capacity. Additionally increasing storage capacity, relative to mean rainfall and evaporation, leads to an increased coefficient of skewness and coefficient of kurtosis indicating a more strongly peaked, fatter tailed pdf. This suggests both an increased likelihood of relatively (a)

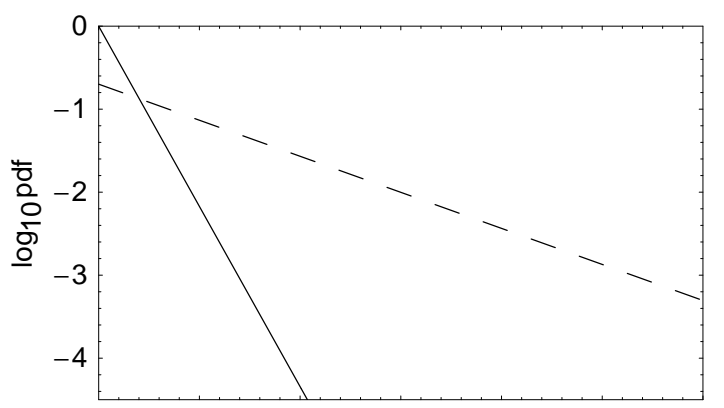

(b)

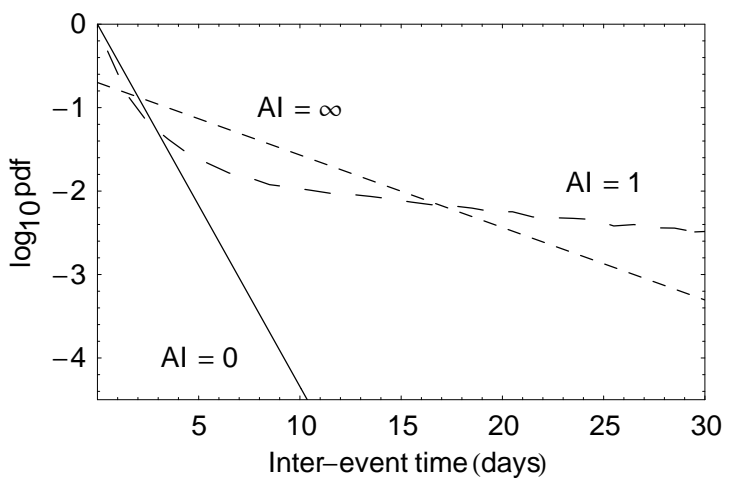

Fig. 2. Conceptual description of the relationship between the rainfall inter-event time (IET) probability density (pdf) and the IET pdf for (a) infiltration excess and (b) saturation excess. For (a) the dashing corresponds to rainfall (solid) and infiltration-excess (dashed) with $I_{\xi} / \bar{I}_{\max }=5$ and $\bar{t}_{b}=1$ day. For (b) dashing corresponds to rainfall (continuous), an aridity index $A I=0$ (also continuous), $A I=1$ (large dashes) and an $A I=\infty$ (small dashes) with $\bar{t}_{b}=1$ day, and $\alpha=5$. Inter-event time pdf estimated at $A I=1$ from a continuous simulation of $10^{5}$ saturation excess events using Eq. (5) 
(a)

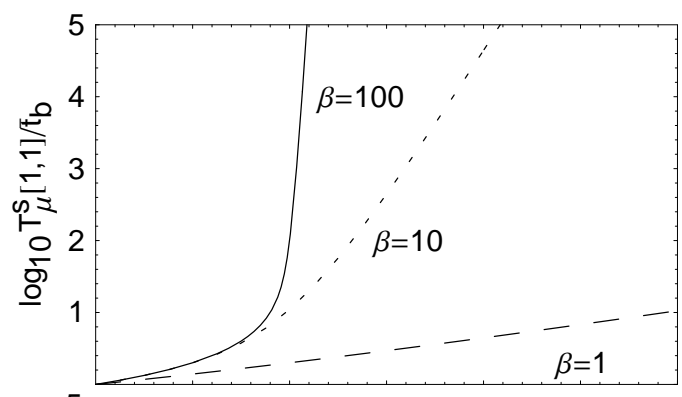

(b)
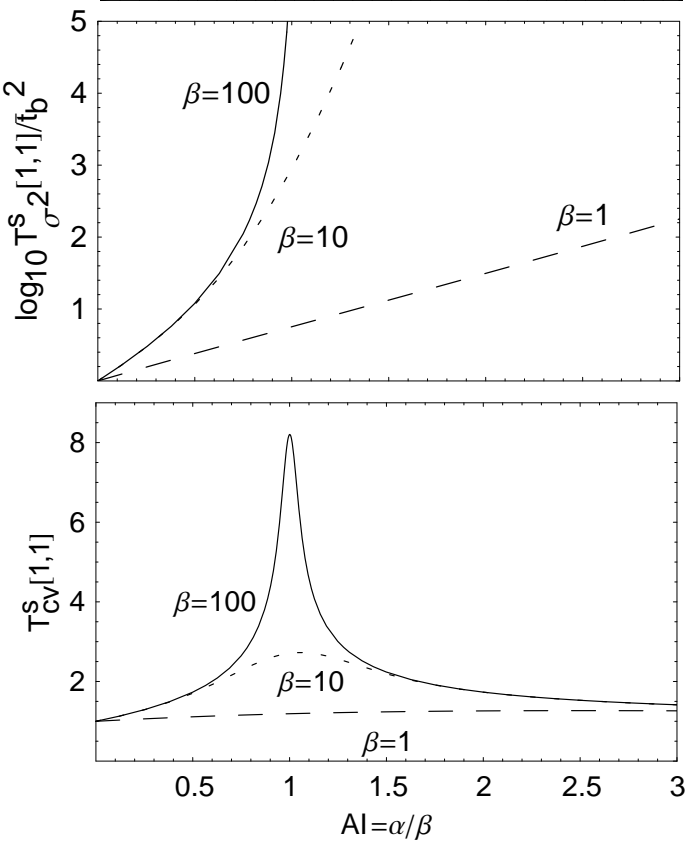

(c)

Fig. 3. Variation of statistics of the storage threshold inter-event time (IET) with aridity index $A I$ showing (a) mean $T_{\mu}^{S}[1,1]$ IET normalised bythe mean inter-storm duration $\bar{t}_{b}$ (Eq. 17) (b) variance $T_{\sigma^{2}}^{s}[1,1]$ of the IET normalised by $\bar{t}_{b}{ }^{2}$ (Eq. 18) and (c) coefficient of variation $T_{c v}^{s}[1,1]$ of the IET (by combining Eq. 17 and Eq. 18) for constant $\beta=1,10$, and 100 (dashed, dotted and continuous respectively).

short IETs (increased peakiness) while at the same time an increased likelihood of much longer IETs (more skewed and fatter tailed). Such behaviour is characteristic of our earlier definition of temporal clustering. For clarity and brevity we restrict further discussion to the first two moments only, allowing us to describe the average IET, the variability as well as the temporal clustering by the $\mathrm{CV}$.

The mean and the variance of the event magnitude decrease, and the coefficient of variation increases with increasing storage capacity (larger $\alpha$ ), or equal decreases in mean storm depth and mean inter-storm evaporation (see Table 2). For the example described above the mean, variance and coefficient of variation of the event magnitude are equal to $Q_{\mu}=0.33 \mathrm{~mm} Q_{\sigma^{2}}=1.2 \mathrm{~mm}^{2}$ and $Q_{c v}=3.3$ respectively. (a)

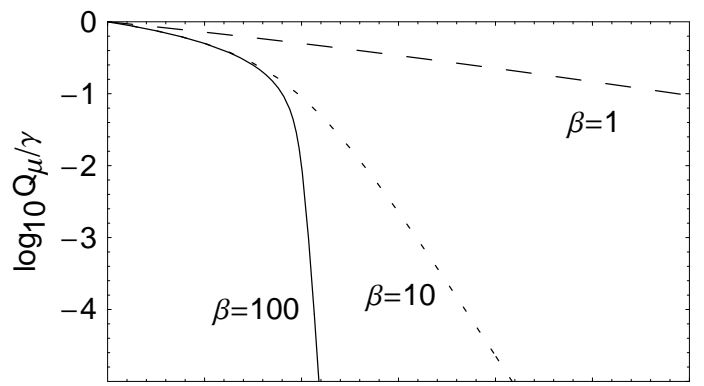

(b)
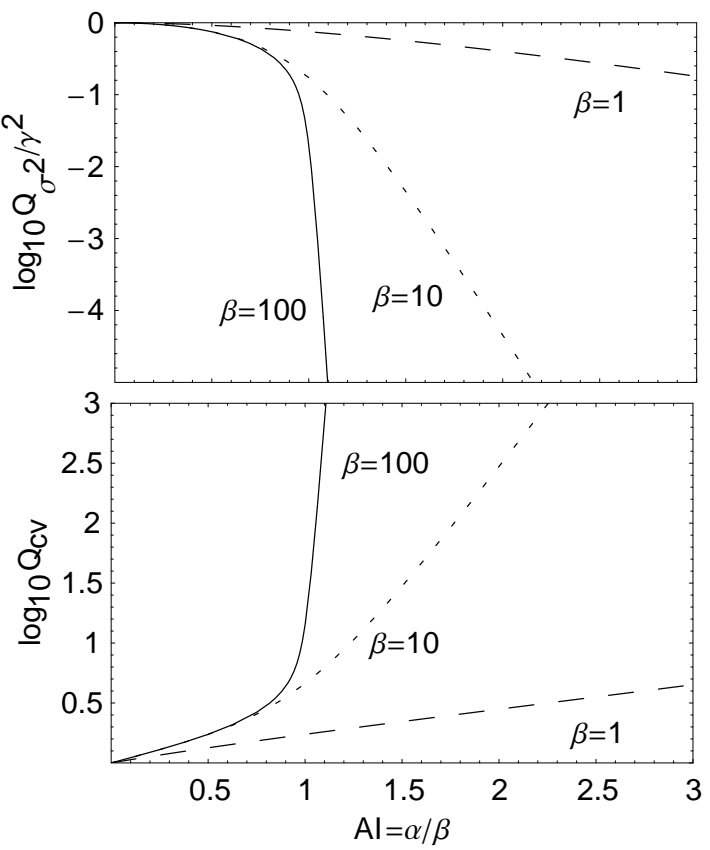

(c)

Fig. 4. Variation of statistics of the storage threshold magnitude with aridity index showing (a) mean $Q_{\mu}$ event magnitude normalised by the mean storm depth $\bar{h}$ (Eq. 12) (b) variance $Q_{\sigma^{2}}$ of the event magnitude normalised by $\bar{h}^{2}$ (Eq. 15) and (c) coefficient of variation $Q_{c v}$ of event magnitude (by combining Eq. 12 and Eq. 15) for constant $\beta=1,10$, and 100 (dashed, dotted and continuous respectively).

\subsubsection{All climates $0<A I<\infty$}

The statistics for the limiting climates described above indicate that the mean and the variance of the IET tend to increase, and the mean and variance of the event magnitude tend to decrease with increasing aridity. However, the variability of event timing relative to the mean $\left(T_{c v}^{s}[1,1]\right)$ tends to peak at intermediate aridity, while the relative variability of event magnitudes continues to increase with increasing aridity. Figures 3 and 4 summarises how the statistics of saturation excess IETs and event magnitudes change as a function of aridity for various levels of the demand ratio.

Strong demand (small $\beta$ ) results in a higher mean and variance but a low $T_{c v}^{s}[1,1]$ and as a result more regular, less clustered events, with a maximum $T_{c v}^{s}[1,1]$ well into in 
the arid region (see Fig. 3). Strong demand also results in a higher mean and variance of the event magnitude at the same aridity, but they tend to decrease as the aridity increases (see Fig. 4). While the mean and variance decrease with increasing aridity, the $\mathrm{CV}$ of the event magnitude increases.

The lower the demand, relative to the storage capacity (larger $\beta$ ), the larger the mean and variance of the IET and the greater the tendency for temporal clustering of events (larger $\left.T_{c v}^{s}[1,1]\right)$. The tendency for saturation excess events to cluster in time is most pronounced in deep stores when supply equals demand i.e. $A I=1$. This is consistent with the observation by Milly (2001) that as the storage capacity increases the maximum variance in soil moisture tends to peak nearer $A I=1$.

These results are at least qualitatively consistent with observations of decreasing mean annual runoff with aridity (Budyko, 1974) and an increasing coefficient of variation of annual runoff with a reduction in mean annual rainfall (Potter et al., 2005). Temporal clustering has been observed in the flood record (Franks and Kuczera, 2002; Kiem et al., 2003) however, this has been attributed to interactions between the Inter-decadal Pacific Oscillation and the El Níno Southern Oscillation changing rainfall patterns. Our quantification of temporal clustering here is based upon on a stationary model of climate. We have as yet found no literature quantifying saturation excess in terms of its temporal dynamics with which we can compare to the statistics derived here.

In summary our results suggest the following about threshold storage triggering: In very arid climates the relative variability of the timing of events is low, as is the contribution of saturation excess to the water balance, as evidenced by a low mean event magnitude. However, the relative variability of the event magnitude $Q_{c v}$ is high; Saturation excess events in semi-arid environments appear to be prone to high coefficients of variation in both the magnitude of events and the time between events, while contributing a non-negligible proportion of the overall water balance; Sub-humid climates have a large proportion of rainfall converted to runoff. The magnitude of these events occur with a lower $Q_{c v}$ than semiarid climates and temporal clustering may be a significant feature of the dynamics; In humid climates storage threshold events contribute a significant proportion of the water balance and the variability (relative to the mean) of the timing and magnitude of these events is low.

\subsection{Frequency magnitude relationships}

It is often the case that we can only observe directly the triggering of events but not the flux. For example the occurrence of soil moisture above a critical value may indicate that macropores are required to have been filled and as a result preferential flow triggered. However, it is typically not possible to measure the flux through either the soil matrix or the macropores, but only relative changes in storage. The timing of triggering and the magnitude of the events are re-

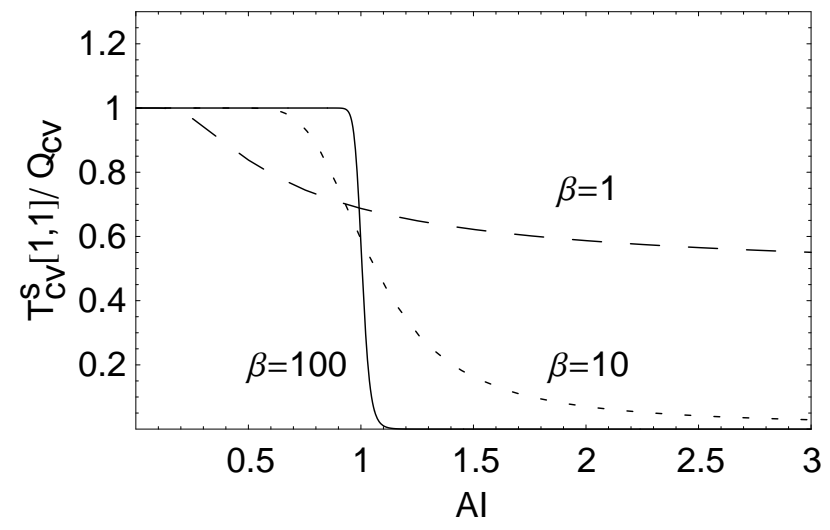

Fig. 5. Ratio of the coefficient of variation of event timing $T_{c v}[1,1]$ and the coefficient of variation of the event magnitude $Q_{c v}$ as a function of aridity. Dashing corresponds to constant $\beta=1$ (large dashed), $\beta=10$ (dotted) and $\beta=100$ (continuous).

lated to one another through soil moisture storage. Therefore in this section, we investigate the relationships between the statistics of event timing and event magnitude.

\subsubsection{Comparison of means}

One would expect that the more frequently threshold storage events occur the greater the contribution of saturation excess to the overall water balance. In fact for the threshold storage model the dimensionless mean saturation excess event magnitude equals the dimensionless mean saturation excess frequency i.e. $Q_{\mu} / \bar{h}=\bar{t}_{b} / T_{\mu}^{s}[1,1]$ as shown by comparing Eq. (12) and Eq. (17). This makes physical sense, for example when $T_{\mu}^{s}[1,1]=\infty, Q_{\mu}$ must be zero, and when $\bar{t}_{b}=T_{\mu}^{s}\left[s_{0}, 1\right], Q_{\mu}$ must equal $\bar{h}$. Noting that $\bar{N}=T_{\mu}^{s}[1,1] / \bar{t}_{b}$ describes the mean number of storms in the IET, it can be shown using Eq. (11) that $Q_{\mu} / \bar{h}=\bar{t}_{b} / T_{\mu}^{s}[1,1]$ is equivalent to $E_{a}=\bar{h}(\bar{N}-1) / \bar{N}$. More specifically this indicates that the inputs (rainfall) must be greater than the losses (actual evaporation) in the time between successive events. Intuitively this must be true irrespective of the nature of the loss function $L[s]$. Whether such a relationship between mean event magnitude and mean event frequency should hold for more general loss functions $L[s]$ is yet to be shown.

\subsubsection{Comparison of relative variability}

In addition to the issue of observability mentioned at the beginning of Sect. 6.2 the relationship between the variability of event magnitudes and the variability of the timing of event triggering may have important ecological implications. For example Sher et al. (2004) noted that the temporal variability of resource supply events in arid ecosystems may be as or even more important ecologically than the variability in the 


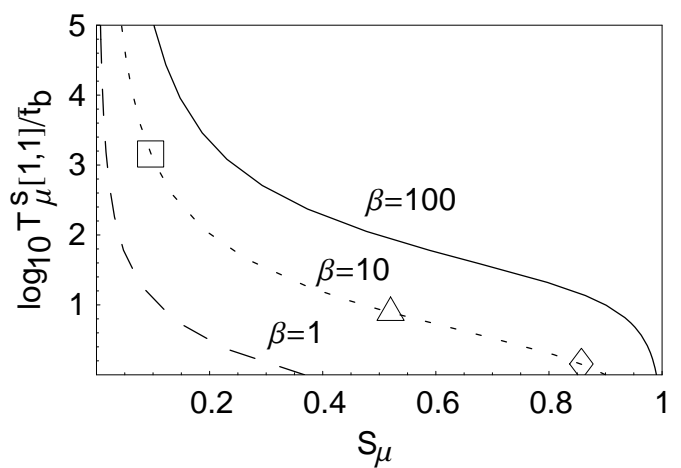

(a)

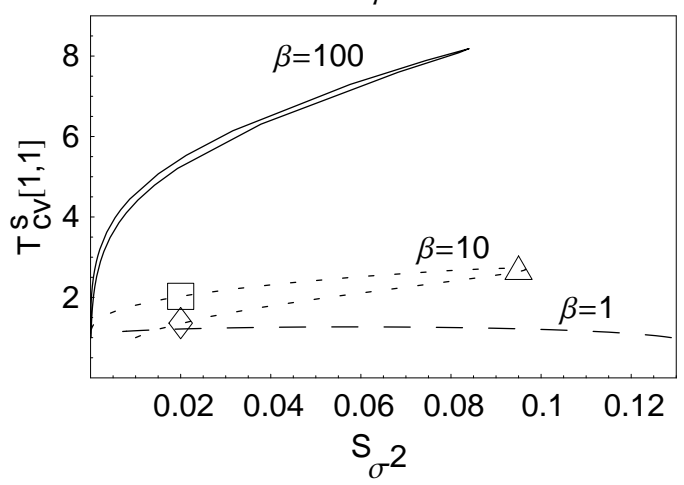

(b)

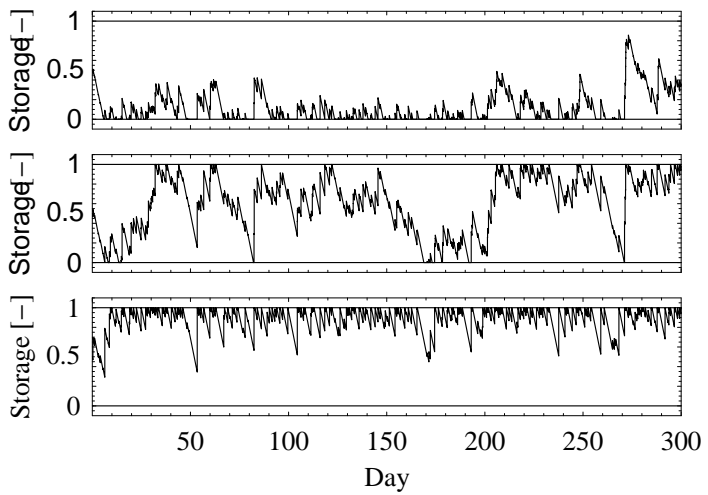

(c)

Fig. 6. Relationship between soil moisture storage and the temporal dynamics showing (a) Mean inter-event time $T_{\mu}^{s}[1,1]$ as a function of mean soil moisture $S_{\mu}$; (b) Coefficient of variation of the interevent time $T_{c v}^{S}[1,1]$ as a function of the variance of soil moisture $S_{\sigma^{2}}$; and (c) Soil moisture time series corresponding to symbols in (a) and (b). Dashing corresponds to constant $\beta=1$ (large dashed), $\beta=10$ (dotted) and $\beta=100$ (continuous). Time series generated as described in Sect. 5.1 with $\bar{t}_{b}=1$ day, $E_{m}=0.5 \mathrm{~mm} /$ day, $w_{0}=5 \mathrm{~mm}$ and $\bar{h}=0.307 \mathrm{~mm}(\square), \bar{h}=0.536 \mathrm{~mm}(\triangle)$, and $\bar{h}=1.66 \mathrm{~mm}(\diamond)$.

magnitude of such events. Here we compare how the CV of IETs relates to the $\mathrm{CV}$ of event magnitude in terms of their ratio $T_{c v}^{s}[1,1] / Q_{c v}$ as a function of aridity (see Fig. 5).

For all climates $T_{c v}^{s}[1,1] \leq Q_{c v}$. In humid climates $T_{c v}^{s}[1,1] \sim Q_{c v}$ meaning event timing and the magnitude of event per storm are both similarly variable with respect to their means. In very arid climates $T_{c v}^{s}[1,1]<Q_{c v}$ indicating less variability in event timing relative to its mean in comparison to event magnitude.
The ratio approaches a step function in the limit of a large storage capacity, relative to the climatic forcing i.e. large $\beta$ (or $\alpha$ not shown), and the step occurs around an aridity index of 1. Mean soil moisture essentially behaves the same way when the storage capacity is large i.e. it is very close to saturation for all $A I<1$ and very close to zero for all $A I>1$ (Milly, 2001). The smaller the storage capacity relative to the climate forcing (small $\beta$ ) the lower the aridity at which $T_{c v}^{s}[1,1]$ and $Q_{c v}$ can be differentiated and the more gradual and smaller the difference with increasing aridity in comparison to larger capacities. Therefore large stores are expected to display much larger flux variability than temporal variability. Despite increased variability in the timing of saturation excess events for deeper stores for sub-humid and semi-arid environments (see Fig. 3), event magnitude variability increases much more rapidly with increasing aridity and continues to do so for $A I>1$.

In terms of the issue of observability our results suggest that the temporal variability of event triggering may give some understanding of the relative variability of event magnitude in humid climates as they are of a similar magnitude in this region. Based upon the relationship between mean event frequency and mean event magnitude the variance of event magnitude per storm event might even be estimated reasonably. In arid climates $T_{c v}^{s}[1,1]$ is much less than $Q_{c v}$ and tells little about the event magnitude variability but none the less provides a reasonably certain measure (i.e. a standard deviation about the same as the mean) of the variability of event timing.

Returning to the hypothesis of Sher et al. (2004), our results suggest that the variability of the timing of potential resource supply events, despite being of long duration on average, is small in comparison to the mean. The temporal variability is also much less than the variability in the magnitude of supply, at least on a per storm basis. This suggests that adaptations by plants and animals to cope with temporal variability may be particularly beneficial in arid climates as it may be a reasonably certain (low variability) component of the hydrological variability.

\subsection{Relationship between temporal statistics and storage}

For some hydrological processes neither the flux nor the triggering are directly observable at the space and time scales at which they occur in the field. This is true in particular for preferential flow. What is measurable, at least at the point scale, is soil moisture storage. Therefore we explore here first how the temporal statistics relate to the statistics of storage and then the sensitivity of the temporal statistics to the initial storage.

\subsubsection{Triggering and soil moisture variability}

Figure 6a shows the relationship between the dimensionless mean saturation excess IET $T_{\mu}^{s}[1,1]$ and the mean storage 
$S_{\mu}$ for constant evaporative demand (constant $\beta$ ). This can also be seen in the time series of storage Fig. $6 \mathrm{c}$ corresponding to the symbols in Fig. 6a. The mean IET increases nonlinearly as the mean storage decreases, and also increases with increasing storage capacity (increasing values of $\beta$ ). The mean IET is most sensitive to changes in low mean soil moisture. This sensitivity is also high at very high mean soil moisture when the storage capacity is large relative to the climatic forcing (large $\beta$ ).

It is evident by comparing the time series Fig. 6c and Fig. 6a that high mean soil moisture is related to low soil moisture variability and frequent event triggering. Low soil moisture variability is also associated with a low mean soil moisture and infrequent triggering. High variability in soil moisture tends to be associated with intermediate $S_{\mu}$ as there is a greater potential for soil moisture fluctuations to explore the entire capacity. This large variability in soil moisture is also associated with high temporal clustering. It can be seen from the relationship between $T_{c v}^{s}[1,1]$ and the variance of storage $S_{\sigma^{2}}$ (Fig. 6b) that for a constant evaporative demand (constant $\beta$ ), the maximum $T_{c v}^{s}[1,1]$ occurs when $S_{\sigma^{2}}$ is also a maximum. This appears to be true for all but the smallest stores (see $\beta=1$ in Fig. 6b) but in this instance the degree of temporal clustering is low in any case.

\subsubsection{The role of initial storage $s_{0}$}

So far we have largely discussed the controls on the IET, that is the time between successive occurrences of storage at capacity. The analytical derivation of FPT statistics $T_{x}\left[s_{0}, 1\right]$ allows us to determine the rainfall controls on the statistical properties of the time to trigger saturation excess flow for the first time since an arbitrary initial storage $s_{0}$. Figure $1 \mathrm{~b}$ shows this FPT as $\tau_{1}$.

The relevance is best explained by the following analogy. Let us assume that storage in the near surface determines the occurrence of preferential flow in a highly nonlinear, threshold like way. We also know from an experiment that there is a rapid movement of rainfall to groundwater via this mechanism. If we take a measurement of the near surface soil moisture, that measurement is an initial condition relative to the time of measurement. As it turns out our analysis (the FPT statistics) reveals that this state of the system determines our level of certainty about the time till the next preferential flow event. Therefore we now have a measure of risk, the mean and variance of the time till the next pesticide leaching event by preferential flow, on the basis of a single measurement of storage. This risk measure includes our knowledge of the structure of rainfall as well as our uncertainty of the timing and magnitude of rainfall events yet to to come.

Figure 7 shows the effect of initial storage on the mean and the $\mathrm{CV}$ of the time till the next saturation excess triggering. It can be seen that the mean FPT $T_{\mu}\left[s_{0}, 1\right]$ decreases as $s_{0}$ increases towards saturation and is more sensitive to $s_{0}$ at higher soil moisture values. Also, as expected, the mean FPT (a)
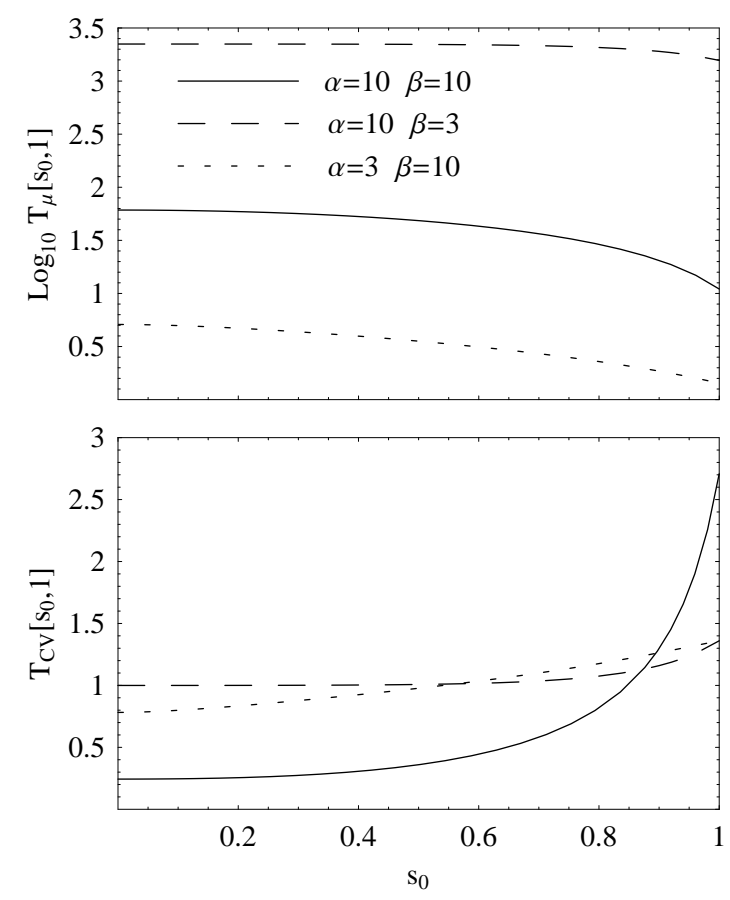

Fig. 7. First passage time statistics vs. the initial soil moisture $s_{0}$. Shown are: (a) the mean $T_{\mu}\left[s_{0}, 1\right]$; and (b) the coefficient of variation $T_{c v}\left[s_{0}, 1\right]$; of the time to reach $s=1$ since an initial soil moisture $s_{0}$.

is longer the higher the aridity (compare ratios of $\alpha$ and $\beta$ in Fig. 7). On the other hand the CV of the FPT $T_{c v}\left[s_{0}, 1\right]$ increases with increasing initial storage. At low $s_{0}$ the more humid the climate the lower $T_{c v}\left[s_{0}, 1\right]$ but this transitions at higher $s_{0}$ such that the more balanced climates and systems with deeper storage capacity, relative to the climatic forcing (larger $\alpha$ and $\beta$ ), tend to have a larger $T_{c v}\left[s_{0}, 1\right]$.

Values of $T_{c v}\left[s_{0}, 1\right]$ when $s_{0}$ is low are $\leq 1$. This can be explained for the case of arid climates where the closer $s_{0}$ is to zero, as well as being close to $S_{\mu}$, only extreme rainfall will trigger an event and for reasons discussed in Sect. 6.1.2 event triggering displays similar but scaled statistical properties to the rainfall. The more humid the climate the further away $s_{0}=0$ is to $S_{\mu}$ and one would expect a more steady, less variable, increase in storage towards capacity and therefore a lower $T_{c v}\left[s_{0}, 1\right]$.

On the other hand when a storage threshold flow event has just occurred $\left(s_{0}=1\right)$ the mean time till the next flow event is a minimum and $T_{c v}\left[s_{0}, 1\right]$ is a maximum. $T_{c v}\left[s_{0}, 1\right]$ is a measure of our uncertainty, relative to our expected value, of the timing of the next storage threshold event due to our uncertainty in the timing and magnitude of rainfall. This implies that if a SE event has just occurred, while we can expect a second event to occur sooner than at any other time, our ability to predict when that will be, relative to the mean time, is actually at its poorest. The high variability of IETs when 
the initial soil moisture is at saturation we believe is due to the greater potential for both much longer periods between events, as a result of the greater potential for drying, as well as a high potential for shorter IETs due to the high potential for event triggering when storage is near capacity.

The variability of threshold hydrological processes seems sensitive to an initial storage near a threshold. Zehe and Blöschl (2004) also found the variability of modelled plot and hillslope scale runoff to be highest when initial soil moisture was at a threshold. In their case they considered a single prescribed rainfall event but multiple realisations of subscale spatial variability of initial soil moisture in relation to the spatially averaged initial soil moisture. When the spatially averaged soil moisture was at the threshold between matrix flow and preferential flow, the variability of modelled runoff was at its greatest. $T_{c v}\left[s_{0}, 1\right]$ is a measure of our uncertainty in the timing of the next event due to our uncertainty of the timing and magnitude of future rainfall events on the basis of a measurement of storage. Additionally the variability in runoff described by Zehe and Blöschl (2004) is a measure of uncertainty in the magnitude of the event due to an uncertain structure of sub-scale soil moisture. Combining these two results it suggests that the time between two consecutive threshold flow events for which the flux is poorly predictable is itself highly uncertain.

\section{Summary and conclusions}

Typically in hydrology we consider the transformation of flux(es) (rainfall, evaporation) to a flux (runoff). Here instead we focus on the transformation of (rainfall) event timing to (flow) event timing. We analytically derived statistics of the temporal dynamics of the flow triggering due to a rainfall intensity threshold and a soil moisture threshold as models for infiltration excess and saturation excess flow mechanisms respectively. The intensity threshold lead to dynamics that did not change the form of the IET pdf. The storage threshold did change the form of the IET pdf leading to temporal clustering of events which tended to peak around an aridity index of one for deep stores.

The mean and the variance of the SE inter-event time were found to increase with increasing climate aridity. The mean and the variance of the SE event magnitude per storm, decreases with increasing aridity, while the CV increases with increasing aridity. It is already established that hillslope saturation excess may dominate in humid climates and to be less significant in arid climates however, the results presented here is the first time that there has been a quantification of the relationships between the temporal structure of event timing, the magnitude of events and the storage across climate gradients.

While the two mechanisms explored are overly simplistic descriptions of real world processes, the results from these two extreme triggers suggest that the actual temporal pattern of triggering will display a mixture of the unclustered and clustered dynamics observed here. There is also a need to better identify and predict thresholds which govern some processes. For example Lehmann et al. (2006, this issue) suggest that the threshold storm amount, governing pipe flow in steep forested hillslopes, may be an emergent property of the connectivity of zones of transient saturation at the soil/bedrock interface. However, there is currently little understanding of what determines the magnitude of these thresholds at different sites (Uchida et al., 2005).

For analytical tractability we have neglected naturally temporally clustered rainfall that may occur at event to interannual time scales (Menabde and Sivapalan, 2000; Franks and Kuczera, 2002). The impact of temporally clustered rainfall we expect will depend upon the degree of memory in the system which would be parameterised by the magnitude of a modified $\beta$ term. Systems with a small $\beta$ would have little memory of long rainfall IETs, while a large $\beta$ would have the potential to "remember" the clustered nature of rainfall. The results presented here will likely be significantly modified when considering climates with strong seasonality and this requires further investigation.

From the frequency-magnitude relationships we found that the dimensionless mean SE event magnitude was equal to the dimensionless mean event frequency and the coefficient of variation of SE event magnitude was found to be always greater than, or at least equal to, the CV of the IET. While no aridity relationships were established for IE, it may be possible to evaluate the contribution of IE as a deviation from the frequency-magnitude relationships derived here. Finally we also established inter-relationships between storage and timing, with a peak in the variance of soil moisture reflecting the peak in temporal clustering of events.

Validation of the results presented here was beyond the scope of this paper. The generality of this work provides the means to develop hypotheses and test the many assumptions with numerical and empirical studies. The question of what to measure and how will depend upon the process under consideration but ideally simulataneous measurments of event based flux, timing and storage in the context of the climate are required to further develop these ideas. Re-analysis of existing threshold phenomena in the context of the temporal dynamics of the process and rainfall may provide an alternative. Such data may include long or ensemble records of overland flow, stream sediment dynamics, pesticide leaching, and pipe flow for example. 


\section{Appendix A}

\section{Derivation of saturation excess temporal statistics}

The integral equation for the pdf of FPTs, $g_{T}$, for processes like the saturation excess one described in this paper was given by (Laio et al., 2001) as:

$$
\begin{aligned}
\frac{\partial g\left(t \mid s_{0}\right)}{\partial t}=-L\left[s_{0}\right] & \frac{\partial g\left(t \mid s_{0}\right)}{\partial s_{0}}-\frac{g\left(t \mid s_{0}\right)}{\bar{t}_{b}} \\
& +\frac{1}{\bar{t}_{b}} \int_{s_{0}}^{s_{\xi}} f_{H}\left[z-s_{0}\right] g(t \mid z) \mathrm{d} z
\end{aligned}
$$

where $L\left[s_{0}\right]\left[\mathrm{T}^{-1}\right]$ is the rate of losses from storage at an initial storage $s_{0}$, at time $t=0, f_{H}[-]$ is the pdf of storm depths normalised by the storage capacity, $z$ is a dummy variable of integration, and $s \xi$ is an arbitrary threshold soil moisture. The raw moments of the FPT are by definition:

$$
T_{n}^{s}\left[s_{0}, s_{\xi}\right]=\int_{0}^{\infty} t^{n} g_{T}\left(t \mid s_{0}\right) \mathrm{d} t
$$

Eq. (A2) motivates us to generalise the derivation to higher order moments by multiplying Eq. (A1) by $t^{n}$, instead of $t$ to just get the mean as done by Laio et al. (2001). So multiplying Eq. (A1) by $t^{n}$, assuming normalised storm depths are exponentially distributed and integrating by parts the time derivative and substituting $T_{n}^{s}\left[s_{0}, s_{\xi}\right]$, results in the following integro-differential equation for the FPT moments:

$$
\begin{aligned}
-n T_{n-1}^{s}\left[s_{0}, s_{\xi}\right]= & -L\left[s_{0}\right] \frac{\mathrm{d} T_{n}^{s}\left[s_{0}, s_{\xi}\right]}{\mathrm{d} s_{0}}-\frac{T_{n}^{s}\left[s_{0}, s_{\xi}\right]}{\bar{t}_{b}}+ \\
& \frac{\alpha}{\bar{t}_{b}} \int_{s_{0}}^{s_{\xi}} e^{-\alpha\left(z-s_{0}\right)} T_{n}^{s}\left[z, s_{\xi}\right] \mathrm{d} z \quad \text { (A3) }
\end{aligned}
$$

Integrating by parts the integral term in Eq. (A3), differentiating the entire equation with respect to $s_{0}$, and substituting for the integral term by rearranging Eq. (A3), leads to a second order ordinary differential equation for the FPT moments $T_{n}^{s}$ :

$$
\begin{aligned}
L\left[s_{0}\right] \frac{\mathrm{d}^{2} T_{n}^{s}}{\mathrm{~d} s_{0}^{2}}+\frac{\mathrm{d} T_{n}^{s}}{\mathrm{~d} s_{0}}\left(\frac{\mathrm{d} L\left[s_{0}\right]}{\mathrm{d} s_{0}}-\right. & \left.\alpha L\left[s_{0}\right]+\frac{1}{\bar{t}_{b}}\right) \\
& =\frac{\mathrm{d} T_{n-1}^{s}}{\mathrm{~d} s_{0}}-n \alpha T_{n-1}^{s}
\end{aligned}
$$

The general solution to Eq. (A4) is given by:

$$
T_{n}^{S}\left[s_{0}, s_{\xi}\right]=C_{2}+C_{1} \int_{1}^{s_{0}} B_{1}[w, 1] \mathrm{d} w+\int_{1}^{s_{0}} B_{2}[w, 1] \mathrm{d} w
$$

where:

$$
\ln \left(B_{1}[a, b]\right)=\alpha(a-1)+\int_{a}^{b} \frac{1}{L[x]}\left(\frac{1}{\bar{t}_{b}}+\frac{\mathrm{d} L[x]}{\mathrm{d} x}\right) \mathrm{d} x
$$

Table A1. Relationships between raw and central moments.

\begin{tabular}{ll}
\hline Central moment & \multicolumn{1}{c}{ Relation to raw moments } \\
\hline Mean & $T_{\mu}=T_{1}$ \\
Variance & $T_{\sigma^{2}}=T_{2}-T_{1}^{2}$ \\
Coefficient of skewness & $T_{\varepsilon}=\frac{T_{3}-3 T_{1} T_{2}+2 T_{1}^{3}}{\left(T_{2}-T_{1}^{2}\right)^{3 / 2}}$ \\
Kurtosis excess & $T_{\kappa}=\frac{T_{4}-4 T_{1} T_{3}+6 T_{1}^{2} T_{2}-3 T_{1}^{4}}{\left(T_{2}-T_{1}^{2}\right)^{2}}-3$ \\
\hline
\end{tabular}

and

$$
\begin{aligned}
B_{2}[w, 1]=- & B_{1}[w, 1] \int_{w}^{1} \frac{n B_{1}[1, y]}{L[y]} \times \\
& \left(\alpha T_{n-1}\left[y, s_{\xi}\right]-\frac{\mathrm{d} T_{n-1}\left[y, s_{\xi}\right]}{\mathrm{d} y}\right) \mathrm{d} y
\end{aligned}
$$

and $w, x$ and $y$ are dummy variables of integration. Two boundary conditions are required to solve for the coefficients $C_{1}$ and $C_{2}$. The first boundary condition is derived when the process begins at the threshold (Masoliver, 1987; Laio et al., 2001) and is obtained by substituting $s_{0}=s_{\xi}$ in Eq. (A3) to get:

$$
\begin{array}{r}
\left.L\left[s_{\xi}\right] \frac{\mathrm{d} T_{n}^{s}\left[s_{0}, s_{\xi}\right]}{\mathrm{d} s_{0}}\right|_{s_{0}=s_{\xi}}=n T_{n-1}^{s}\left[s_{\xi}, s_{\xi}\right]+ \\
\frac{T_{n}^{s}\left[s_{\xi}, s_{\xi}\right]}{\bar{t}_{b}}
\end{array}
$$

The second boundary condition is required to describe the time to reach the threshold having begun at the lower boundary and is obtained from substitution of $s_{0}=0$ in Eq. (A3) (Masoliver, 1987; Laio et al., 2001) resulting in:

$$
\begin{aligned}
-n T_{n-1}^{s}\left[0, s_{\xi}\right]=- & \frac{T_{n}^{s}\left[0, s_{\xi}\right]}{\bar{t}_{b}}+ \\
& \frac{\alpha}{\bar{t}_{b}} \int_{0}^{s \xi} e^{-\alpha\left(z-s_{0}\right)} T_{n}^{s}[z, s \xi] \mathrm{d} z
\end{aligned}
$$

The coefficient $C_{1}$ can be obtained by differentiating Eq. (A5) with respect to $s_{0}$, inserting this into Eq. (A8), substituting $s_{0}=s \xi$ and then solving for $C_{1}$, which is dependent upon $C_{2}$. Substituting Eq. (A5) in the second boundary condition Eq. (A9) gives a second equation for $C_{1}$ also dependent upon $C_{2}$. Equating these two expressions and solving for $C_{2}$ gives:

$$
\begin{aligned}
C_{2} & =\left(\frac{B_{3}[3]+n T_{n-1}\left[0, s_{\xi}\right] \bar{t}_{b}}{B_{3}[1]}-\right. \\
& \left.\frac{B_{4}[3]-n T_{n-1}[s \xi, s \xi]}{B_{4}[1]}\right) \div\left(\frac{e^{-\alpha s_{\xi}}}{B_{3}[1]}+\frac{1}{\bar{t}_{b} B_{4}[1]}\right)
\end{aligned}
$$


where

$$
B_{4}[A]=L\left[s_{\xi}\right] B_{A}[s \xi, 1]-\frac{1}{\bar{t}_{b}} \int_{s_{0}}^{1} B_{A}[w, 1] \mathrm{d} w
$$

and

$$
\begin{array}{r}
B_{3}[A]=\int_{0}^{s_{\xi}} \alpha e^{-z \alpha} \int_{1}^{z} B_{A}[w, 1] \mathrm{d} w \mathrm{~d} z+ \\
\int_{0}^{1} B_{A}[w, 1] \mathrm{d} w
\end{array}
$$

The subscript $A$ is a reference to $B_{A}$ as given by Eq. (A6) or Eq. (A7). Substituting Eq. (A10) into one of the original expressions for $C_{1}$ gives:

$$
\begin{gathered}
C_{1}=-\left(n T_{n-1}\left[s_{\xi}, s_{\xi}\right]-e^{\alpha s_{\xi}}\left(n T_{n-1}\left[0, s_{\xi}\right]+\right.\right. \\
\left.\left.\frac{B_{3}[3]}{\bar{t}_{b}}\right)-B_{4}[3]\right) \div\left(\frac{1}{\bar{t}_{b}} e^{\alpha s \xi} B_{3}[1]+B_{4}[1]\right)
\end{gathered}
$$

The central moments can be derived from these raw moments using the relationships described in Table A1 and as discussed in Sect. 5.2.

\section{Appendix B}

\section{List of Symbols}

\begin{tabular}{lll} 
Symbol & Description & Units \\
\hline \multicolumn{2}{l}{ Soil parameters } & \\
$I_{\xi}$ & Infiltration capacity & $\mathrm{L} / \mathrm{T}$ \\
$w_{0}$ & Storage capacity & $\mathrm{L}$ \\
$s$ & Normalised soil moisture storage & - \\
$s_{0}$ & Initial soil moisture & - \\
$s_{\xi}$ & Threshold soil moisture & - \\
Climate & parameters & \\
$h$ & Storm depth & $\mathrm{L}$ \\
$\bar{h}$ & Mean storm depth & $\mathrm{L}$ \\
$t_{b}$ & Inter storm duration & $\mathrm{T}$ \\
$\bar{t}_{b}$ & Mean inter-storm duration & $\mathrm{T}$ \\
$E_{m}$ & Potential evaporation & $\mathrm{L} / \mathrm{T}$ \\
$I_{\max }$ & Max within storm rainfall intensity & $\mathrm{L} / \mathrm{T}$ \\
$\bar{I}_{\text {max }}$ & Mean $I_{\text {max }}$ & $\mathrm{L} / \mathrm{T}$ \\
Probability terms & \\
$f_{X}$ & Probability density & $\mathrm{X}$ \\
$g_{T}$ & First passage time probability density & $\mathrm{T}$ \\
$P[\quad]$ & Probability & - \\
\hline
\end{tabular}

Continued...

${ }^{a}$ Units $\mathrm{X}$ correspond to the random variable

\begin{tabular}{llc} 
Symbol & Description & Unit \\
\hline$\mu$ & Mean & $\mathrm{X}$ \\
$\sigma$ & Standard deviation & $\mathrm{X}$ \\
$\sigma^{2}$ & Variance & $\mathrm{X}^{2}$ \\
$c v$ & Coefficient of variation & - \\
$\varepsilon$ & Coefficient of skewness & - \\
$\kappa$ & Coefficient of kurtosis & -
\end{tabular}

\section{Dimensionless hydrological parameters and statistics}

$\alpha$

$\beta$

AI

$E_{a}$

$\bar{N}$

$Q_{x}$

$S_{x}$

$T_{x}^{I}\left[I_{\xi}\right]$

$T_{x}^{r}$

Supply ratio

Demand ratio

Aridity index

Mean actual evaporation

Mean number of storms in the IET

Saturation excess event magnitude $a$ statistic

$T_{x}^{s}\left[s_{0}, s_{\xi}\right] \quad$ Statistic of the time to reach $s_{\xi}$ since an $\quad b$ initial soil moisture $s_{0}$

\footnotetext{
${ }^{a}$ Units correspond to $Q_{\mu}[\mathrm{L}], Q_{\sigma^{2}}\left[\mathrm{~L}^{2}\right]$, and $Q_{c v}[-]$

${ }^{b}$ Units correspond to $T_{\mu}[\mathrm{T}], \mathrm{T}_{\sigma^{2}}\left[\mathrm{~T}^{2}\right], T_{c v}[-], T_{\varepsilon}[-]$, and $T_{\kappa}[-]$
}

Acknowledgements. The research was made possible by an Australia Postgraduate Award (Industry) from the Australian Research Council in conjunction with the Centre for Groundwater Studies. In addition GSM would like to thank A. Porporato for his assistance and comments on an early manuscript.

Edited by: G. Hancock

\section{References}

Arora, V.: The use of the aridity index to assess climate change effect on annual runoff., J. Hydrol., 265, 164-177, 2002.

Bauters, T.: Soil water content dependent wetting front characteristics in sands., J. Hydrol., 231-232, 244-254, 2000.

Beven, K. and Germann, P.: Macropores and water flow in soils., Water Resour. Res., 18, 1311-1325, 1982.

Budyko, M.: Climate and Life., Academic Press, New York, 1974.

Crockford, R. and Richardson, D.: Partitioning of rainfall into throughfall, stemflow and interception : effect of forest type, groundcover and climate, Hydrol. Process., 14, 2903-2920, 2000.

Dekker, L. W., Doerr, S. H., Oostindie, K., Ziogas, A. K., and Ritsema, C. J.: Water repellency and critical soil water content in a dune sand, Soil Sci. Soc. Am. J., 65, 1667-1674, 2001.

Dunne, T.: Field studies of hillslope flow processes, in: Hillslope Hydrology, (edited by: M. J. Kirkby), John Wiley and Sons, Chichester, West Sussex, UK, 1978. 
Fitzjohn, C., Ternan, J., and Williams, A.: Soil moisture variability in a semi-arid gully catchment: implications for runoff and erosion control., Catena, 32, 55-70, 1998.

Fortin, J., Gagnon-Bertrand, E., Vezina, L., and Rompre, M.: Preferential bromide and pesticide movement to tile drains under different cropping practices, J. Environ. Qual., 31, 1940-1952, 2002.

Franks, S. W. and Kuczera, G.: Flood frequency analysis: Evidence and implications of secular climate variability, New South Wales, Water Resour. Res., 38, 1062, doi:10.1029/2001WR000 232, 2002.

Godano, C., Alonzo, M., and Vildaro, G.: Multifractal approach to time clustering of earthquakes: Application to Mt. Vesuvio seismicity, Pure and Appl. Geophys., 149, 375-390, 1997.

Haria, A. H., Johnson, A. C., Bell, J. P., and Batchelor, C. H.: Watermovement and isoproturon behavior in a drained heavy clay soil , 1. Preferential flow processes, J. Hydrol., 163, 203-216, 1994.

Heppell, C. M., Worrall, F., Burt, T. P., and Williams, R. J.: A classification of drainage and macropore flow in an agricultural catchment, Hydrol. Process., 16, 27-46, 2002.

Horton, R.: The role of infiltration in the hydrologic cycle., Trans. Am. Geophys. Union, 14, 446-460, 1933.

Hyer, K. E., Hornberger, G. M., and Herman, J. S.: Processes controlling the episodic streamwater transport of atrazine and other agrichemicals in an agricultural watershed, J. Hydrol., 254, 4766, 2001.

Kiem, A. S., Franks, S. W., and Kuczera, G.: Multi-decadal variability of flood risk, Geophys. Res. Let., 30, 1035, doi:10.1029/2002GL015 992, 2003.

Kjær, J., Olsen, P., Ullum, M., and Grant, R.: Leaching of Glyphosate and Amino-Methylphosphonic Acid from Danish agricultural field sites., J. Environ. Qual., 34, 608-620, 2005.

Kohler, A., Abbaspour, K. C., Fritsch, M., and Schulin, R.: Using simple bucket models to analyze solute export to subsurface drains by preferential flow, Vadose Zone J., 2, 68-75, 2003.

Kung, K.-J.: Preferential flow in a sandy vadose zone, 2 : Mechanisms and implications., Geoderma, 22, 59-71., 1990.

Laabs, V., Amelung, W., Pinto, A., and Zech, W.: Fate of pesticides in tropical soils of Brazil under field conditions, J. Environ. Qual., 31, 256-268, 2002.

Laio, F., Porporato, A., Ridolfi, L., and Rodriguez-Iturbe, I.: Mean first passage times of processes driven by white shot noise, Physical Rev. E, 6303, 2001.

Lehmann, P., Hinz, C., McGrath, G., Tromp-van Meerveld, H. J., and McDonnell, J. J.: Rainfall threshold for hillslope outflow: an emergent property of flow pathway connectivity., Hydrol. Earth Sys. Sci. Discussions, 3, 2923-2961, 2006.

Masoliver, J.: 1st-Passage times for non-Markovian processes Shot noise, Physical Rev. A, 35, 3918-3928, 1987.

Menabde, M. and Sivapalan, M.: Modeling of rainfall time series and extremes using bounded random cascades and Levy-stable distributions, Water Resour. Res., 36, 3293-3300, 2000.

Milly, P. C. D.: An analytic solution of the stochastic storage problem applicable to soil-water, Water Resour. Res., 29, 3755-3758, 1993.

Milly, P. C. D.: Climate, interseasonal storage of soil-water, and the annual water-balance, Adv. Water Resour., 17, 19-24, 1994.

Milly, P. C. D.: A minimalist probabilistic description of root zone soil water, Water Resour. Res., 37, 457-463, 2001.
Mosley, M.: Streamflow generation in a forested watershed., Water Resour. Res., 15, 795-806, 1976.

Papoulis, A.: Probability, Random Variables and Stochastic Processes, 3rd ed., McGraw-Hill, New York, 2001.

Potter, N. J., Zhang, L., Milly, P. C. D., McMahon, T. A., and Jakeman, A. J.: Effects of rainfall seasonality and soil moisture capacity on mean annual water balance for Australian catchments, Water Resour. Res., 41, W06 007, doi:10.1029/2004WR003 697, 2005.

Ridolfi, L., D’Odorico, P., Porporato, A., and Rodriguez-Iturbe, I.: Duration and frequency of water stress in vegetation: An analytical model, Water Resour. Res., 36, 2297-2307, 2000.

Rodriguez-Iturbe, I. and Isham, V.: Some models for rainfall based on stochastic point processes, Proc. Royal Soc. Lond. Ser. AMath. Phys. Eng. Sci., 410, 269-288, 1987.

Rodriguez-Iturbe, I., Porporato, A., Ridolfi, L., Isham, V., and Cox, D. R.: Probabilistic modelling of water balance at a point: the role of climate, soil and vegetation, Proc. Royal Soc. Lond. Ser. A-Math. Phys. Eng. Sci., 455, 3789-3805, 1999.

Rodriguez-Iturbe, I., Porporato, A., Laio, F., and Ridolfi, L.: Plants in water controlled ecosystems: active role in hydrologic process and response to water stress. I Scope and general outline., Adv. Water. Resour., 24, 695-705, 2001.

Rundle, J. B., Turcotte, D. L., Rundle, P. B., Yakovlev, G., Shcherbakov, R., Donnellan, A., and Klein, W.: Pattern dynamics, pattern hierarchies, and forecasting in complex multi-scale earth systems., Hydrol. Earth Syst. Sci. Discuss., 3, 1045-1069, 2006, http://www.hydrol-earth-syst-sci-discuss.net/3/1045/2006/.

Sher, A., Goldberg, D., and Novoplansky, A.: The effect of mean and variance in resource supply on survival of annuals from Mediterranean and desert environments., Oecologica, 141, 353362, 2004.

Struthers, I., Sivapalan, M., and Hinz, C.: Conceptual examination of climatesoil controls upon rainfall partitioning in an openfractured soil: I. Single storm response, Adv. Water Resour., 30 (3), 505-517, 2007a.

Struthers, I., Sivapalan, M., and Hinz, C.: Conceptual examination of climate soil controls upon rainfall partitioning in an openfractured soil II: Response to a population of storms, Adv. Water Resour., 30 (3), 518-527, $2007 \mathrm{~b}$.

Teich, M., Heneghan, C., Lowen, S., Ozaki, T., and Kaplan, E.: Fractal character of the neural spike train in the visual system of the cat, J. Opt. Soc. Am. A, 14, 529-545, 1997.

Tromp-van Meerveld, H. and McDonnell, J.: Threshold relations in subsurface stormflow 1. A 147 storm analysis of the Panola hillslope., Water Resour. Res., 42, 2006.

Uchida, T., van Meerveld, I. T., and McDonnell, J.: The role of lateral pipe flow in hillslope outflow response: an intercomparison of non-linear hillslope response., J. Hydrol., 311, 117-133, 2005.

Wang, Z., Feyen, J., and Ritsema, C.: Susceptibility and predictability of conditions for preferential flow., Water Resour. Res., 34, 2183-2190, 1998.

Whipkey, R.: Subsurface stormflow from forested slopes., Bull. Int. Assoc. Sci. Hydrol., 10, 74-85, 1965.

Wood, M. A., Simpson, P. M., Stambler, B. S., Herre, J. M., Bernstein, R. C., and Ellenbogen, K. A.: Long-term temporal patterns of ventricular tachyarrhythmias., Circulation, 91, 23712377, 1995. 
Zehe, E. and Blöschl, G. N.: Predictability of hydrologic response at the plot and catchment scales: Role of initial conditions, Water Resour. Res., 40, W10 202, doi:10.1029/2003WR002 869, 2004.
Zeng, N., Shuttleworth, J., and Gash, J.: Influence of temporal variability of rainfall on interception loss. Part I. Point analysis., J. Hydrol., 228, 228-241, 2000. 